\title{
Establishment and Characterization of FusionRed Stable Transfected Canine Prostate Adenocarcinoma and Transitional Cell Carcinoma Cells
}

\author{
SUHAYLA ALNAJJAR ${ }^{1,2}$, INGO NOLTE ${ }^{1}$, JAN TORBEN SCHILLE ${ }^{1,2}$, SINA SENDER $^{2}$, \\ NARES TRAKOOLJU ${ }^{3}$, SIMON VILLA PEREZ ${ }^{2}$, DIETMAR ZECHNER ${ }^{4}$, BRIGITTE VOLLMAR ${ }^{4}$, \\ CHRISTIAN JUNGHANSS ${ }^{2}$ and HUGO MURUA ESCOBAR ${ }^{2,5}$ \\ ${ }^{1}$ Small Animal Clinic, University of Veterinary Medicine Hannover, Hannover, Germany; \\ ${ }^{2}$ Division of Medicine, Haematology, Oncology and Palliative Medicine, University of Rostock, Rostock, Germany; \\ ${ }^{3}$ Research Institute for Farm Animal Biology (FBN), Institute of Genome Biology, Dummerstorf, Germany; \\ ${ }^{4}$ Institute for Experimental Surgery, University of Medicine Rostock, Rostock, Germany; \\ ${ }^{5}$ Comprehensive Cancer Center MV, Campus Rostock, Rostock, Germany
}

\begin{abstract}
Background/Aim: Cancer cell inoculation is routinely used to evaluate novel therapeutic approaches in vivo. However, without reporter genes enabling deep tissue imaging, study of early tumor progression and therapeutic responses is often limited. We describe the establishment and characterization of two canine cancer cell lines stably expressing red fluorescence proteins as tools for later in vivo imaging. Materials and Methods: Two red fluorescence cell lines were generated by plasmid transfection. Fluorescence protein expression was confirmed by flow cytometry and microscopy. Deep tissue imaging was demonstrated in mice using a NightOWL LB 983. Gene expression changes after transfection were analyzed by RNAseq. Results: Both cell lines were detectable in vivo by subcutaneous injection of $1 \times 10^{6}$ cells. RNAseq revealed up to 2005 transfection-induced differentially expressed genes but no significant changes in cellular key pathways. Conclusion: The fluorescent cell lines provide a solid basis for future in vivo studies on canine cancer.
\end{abstract}

Advanced experimental approaches in cancer research require the establishment of tumor-specific in vivo animal

This article is freely accessible online.

Correspondence to: Hugo Murua Escobar, Department of Internal Medicine, Medical Clinic III, Clinic for Hematology, Oncology and Palliative Care, University Medical Center Rostock, Rostock, Germany. Tel: +49 3814947519, Fax: +49 3814945898, e-mail: Hugo.Murua.Escobar@med.uni-rostock.de

Key Words: Prostate cancer, red fluorescent protein, in vitro and in vivo imaging. models. Therefore, cell lines represent a powerful tool allowing the generation of neoplasia mimicking closely the initial tumors through xenografts $(1,2)$. Within these models, the characterization of tumor development and the possibility to monitor tumor cell migration is of major interest for the evaluation of novel therapeutics. Fluorescent proteins and bioluminescent systems provide unique opportunities for non-invasive labeling and tracking of specific cell types in living organisms in real-time.

Together with the possibilities of whole-body imaging, fluorescent proteins can be used to visualize many types of cancer processes, including primary tumor growth, tumors cell motility and invasion, metastases, and tumor-host interaction (3-5). For deep imaging of animal tissues, light penetration can be achieved using near-infrared wavelengths, which are less absorbed by tissue, and require proteins with emission spectra in the red and far-red wavelengths $(3,5-9)$. Tumor cell lines stably expressing detectable fluorescent proteins enable evaluation of the efficacy of therapeutic anticancer treatments through whole-body imaging without the need for further invasive procedures in tumor-bearing xenograft models.

However, stable integration of transfected plasmids bears the risk of insertional mutagenesis potentially affecting genomic stability and gene expression and thereby potentially altering the cellular behavior of the transfected cell lines (10). Therefore, a comprehensive comparative molecular analysis of transfected and native cell lines is crucial to identify concordant and discordant biological functions, processes, and pathways. Next generation sequencing of RNA has revolutionized the ability to characterize cancers at the genomic, transcriptomic, and epigenetic levels (11). 
Compared to conventional approaches, RNA sequencing technologies are more specific and sensitive, and allow the analysis of transcriptomes from minimal amounts of sampling material. Comparable to humans, dogs naturally develop prostate cancer (12). Histopathologically, prostate cancer can be classified into prostate adenocarcinoma (PAC) or transitional cell carcinoma (TCC) occurring in the bladder neck, urethra or periurethral duct (13-15). Studies showed that PAC is likely to infiltrate the closely neighbored bladder-neck and likewise, TCC also infiltrates prostatic tissue, therefore, differentiation between PAC and TCC in prostatic tumors is recommended $(13,16)$.

Human and canine prostate carcinomas are at present incurable once they have metastasized. Canine prostate cancer is considered a reliable model for the testing of novel therapies for castrate-resistant prostate cancer in humans (15) and the histologic and biologic characteristics of TCC in dogs are almost similar to those of TCC in humans (17). Consequently, data obtained in dogs can be an important step for the progress in human tumor therapy. Since metastases are highly resistant to current conventional therapies, new therapeutic strategies are necessary.

This study describes the establishment and characterization of two canine tumor cell lines that stably express a red fluorescent protein. The fluorescent cells were detectable in vivo and therefore provide a valuable tool for the evaluation of new therapeutic approaches for canine cancer in vitro and in vivo.

Further, the transcriptome analysis of transfected cell lines in comparison to their parental cells revealed, that the integration of FusionRed did not affect the biological process in the generated cell lines.

\section{Materials and Methods}

Cell lines and culture. Canine transitional cell carcinoma cell line TihoDProCarc/TCC0840 (0840) and prostate adenocarcinoma cell line TihoDProAdCarc0846 (0846) were previously derived by our group from canine prostate carcinomas. 0840 was generated from a 10-year-old castrated Pit Bull Terrier $(18,19)$ and 0846 was generated from a 6.3-year-old intact German Rough Haired Pointer (20). Both cell lines have been shown to express CLDN3, -4, and 7 (21). Cells were cultivated separately in $25 \mathrm{~cm}^{2}$ cell culture flasks in medium 199 (Gibco by Life Technologies, Darmstadt, Germany) supplemented with $10 \%$ Fetal Bovine Serum (FBS Superior, Biochrom $\mathrm{GmbH}$, Berlin, Germany) and $2 \%$ penicillin/streptomycin (Biochrom $\mathrm{GmbH}$ ), and incubated in a humidified incubator maintained at $37^{\circ} \mathrm{C}$ with $5 \% \mathrm{CO}_{2}$. The culture medium was replaced twice per week.

Red fluorescence expression plasmid. For transfection, the pFusionRed-C (Evrogen, Moscow, Russia) plasmid was used. The vector encodes for the red fluorescent protein FusionRed as well as a neomycin resistance gene allowing selection of the stably transfected cells using Geneticin ${ }^{\circledR}$ Selective Antibiotic (G418) (Life
Technologies, Darmstadt, Germany). The vector was inserted into E. coli $\mathrm{DH} 5 \alpha$ competent cells according to standard heat shock transformation procedures for further multiplication. Expanded plasmid DNA was extracted from bacteria culture using Nucleo Bond ${ }^{\circledR}$ PC 500 plasmid DNA purification Kit (MACHEREYNAGEL GmbH, Düren, Germany).

Transfection of canine tumor cell lines. Cell lines were seeded with a density of $5 \times 10^{5}$ cells in 6-well plates $24 \mathrm{~h}$ before transfection. Transfection was performed according to the manufacturer's protocol using $6 \mu \mathrm{l}$ X-treme GENE HP DNA Transfection Reagent (Roche GmbH, Mannheim, Germany) in $200 \mu \mathrm{l}$ serum-reduced Opti-MEM I media (Life Technologies) containing $2.5 \mu \mathrm{g}$ of the isolated and purified pFusionRed-C plasmid. The transfection complex remained for $20 \mathrm{~min}$ at room temperature. After adding the transfection complex to the respective cell lines, plates were incubated in a humidified $5 \% \mathrm{CO}_{2}$ incubator for $48 \mathrm{~h}$ at $37^{\circ} \mathrm{C}$. The expression of the fluorescent protein was verified using a Leica DMI 6000B fluorescence microscope (Leica Microsystem GmbH, Wetzlar, Germany).

Geneticin $^{\circledR}$ selective antibiotic kill curve assay. The titration of a suitable amount of antibiotics required for the selection of transfected cell lines was performed using a kill curve assay. Different G418 concentrations (0, 100, 200, 400, 600, 800, 1000 $\mu \mathrm{g} / \mathrm{ml})$ were applied on 100,000 parental cells, seeded in 12-well plates. For the selection of positive cells after transfection, the lowest concentration was chosen in which no non-transfected cell survived after seven days of G418 exposure as determined by microscopy and flow cytometry analysis.

Selection of positively transfected cells. One day after transfection, the culture medium 199 was replaced with medium 199 containing the antibiotic G418. A concentration of $200 \mu \mathrm{g} / \mathrm{ml} \mathrm{G} 418$ was used for both transfected cell lines. Subsequently, the selection medium was changed every $48 \mathrm{~h}$ for the first two weeks, which led to a selection of cells that stably integrated the FusionRed plasmid, including the encoded antibiotic resistance gene, into their genomic DNA. Accordingly, cells not expressing the construct were killed by G418.

Fluorescence microscopy and flow cytometry. During cell selection, cells were observed by fluorescence microscopy weekly. FusionRed expression was quantified in a MACSQuant flow cytometer monthly (MACS Miltenyi Biotech, Bergisch Gladbach, Germany). Cells were detached with Tryp-LE ${ }^{\mathrm{TM}}$ Express (Gibco by Life Technologies), adjusted to $1 \times 10^{6}$ cells in $500 \mu$ phosphate-buffered saline (PBS) and examined using the PE channel in a flow cytometer. Results were analyzed with FlowJo Version 7.6.5 (FlowJo, Ashland, OR, USA). Transfected cell lines were used for all further experiments once they exceeded passage 30 .

In vitro imaging using the NightOWL LB 983 in vivo imaging system. Viable cells were harvested, counted, and plated at a density of $2.5 \times 10^{6}$ per well in a 96 -well plate and followed by a 1:2 serial dilution until reaching $0.156 \times 10^{6}$ cells per well in duplicates. Each well was set to a volume of $250 \mu 1$. Non-transfected cells were used as negative controls in equivalent concentrations. The plate was incubated overnight, and images were taken with the NightOWL LB 983 (Berthold Technologies, Bad Wildbad, Germany). An excitation filter of $525 \mathrm{~nm}$ and an emission filter of $655 \mathrm{~nm}$ with variable 
Table I. List of mice used in animal studies.

\begin{tabular}{lcccc}
\hline Mouse ID & Mouse strain & Fluorescently labeled canine tumor cells & Right flank & Left flank \\
\hline Rag1 & 129S4-Rag2 & DT0840-FusionRed & $1 \times 10^{6}$ cells in PBS/Matrigel & $1 \times 10^{6}$ cells in PBS \\
Rag2 & 129S4-Rag2 & DT0846-FusionRed & $1 \times 10^{6}$ cells in PBS/Matrigel & $1 \times 10^{6}$ cells in PBS \\
Nu1 & NMRI-Foxn1 nu/nu & DT0840-FusionRed & $1 \times 10^{6}$ cells in PBS/Matrigel & $1 \times 10^{6}$ cells in PBS \\
Nu2 & NMRI-Foxn1 nu/nu & DT0846-FusionRed & $1 \times 10^{6}$ cells in PBS/Matrigel & $1 \times 10^{6}$ cells in PBS
\end{tabular}

A

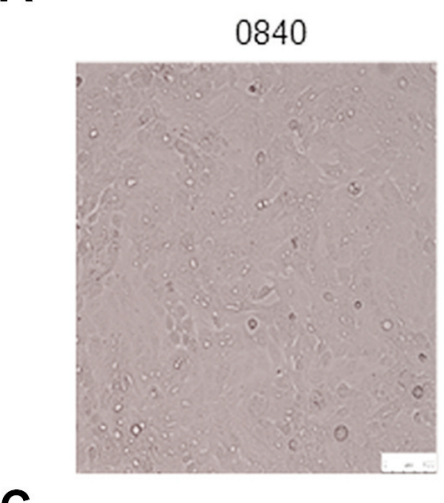

C

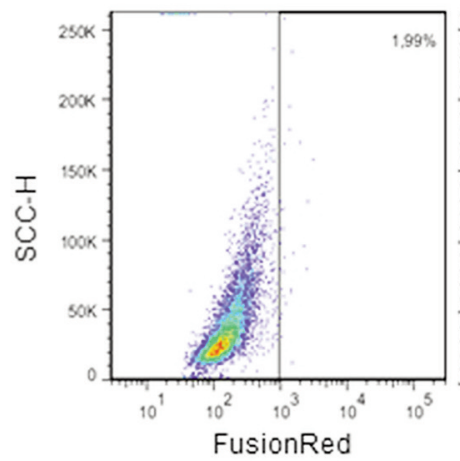

0840 -FusionRed
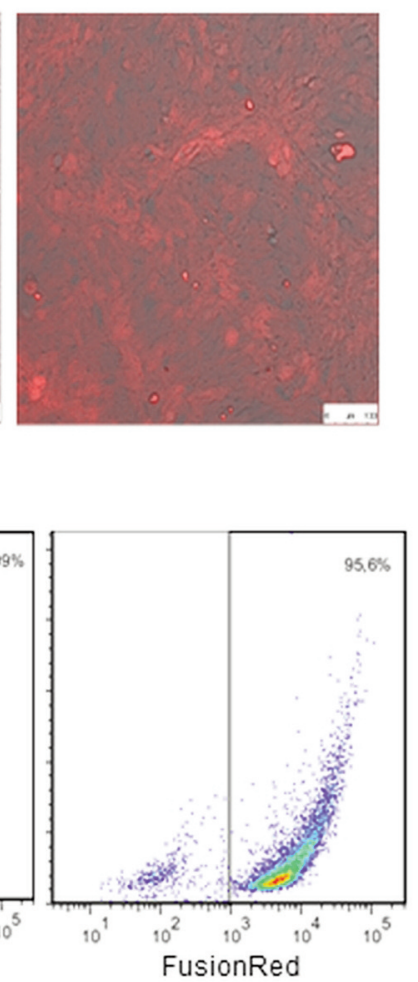

B

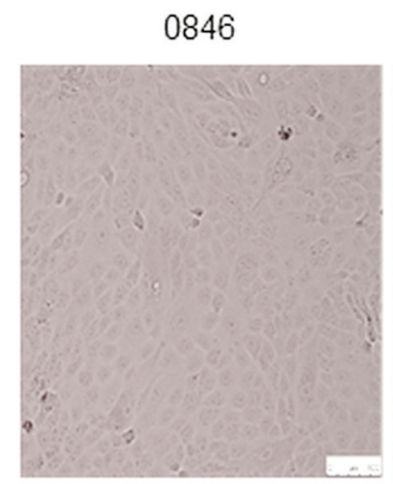
0846 -FusionRed

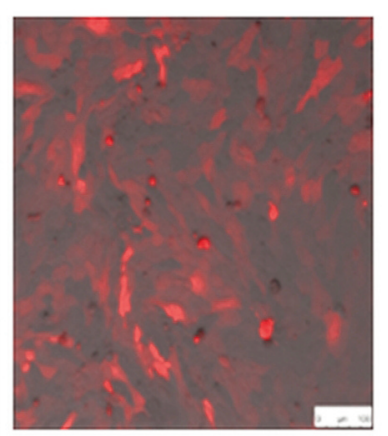

D

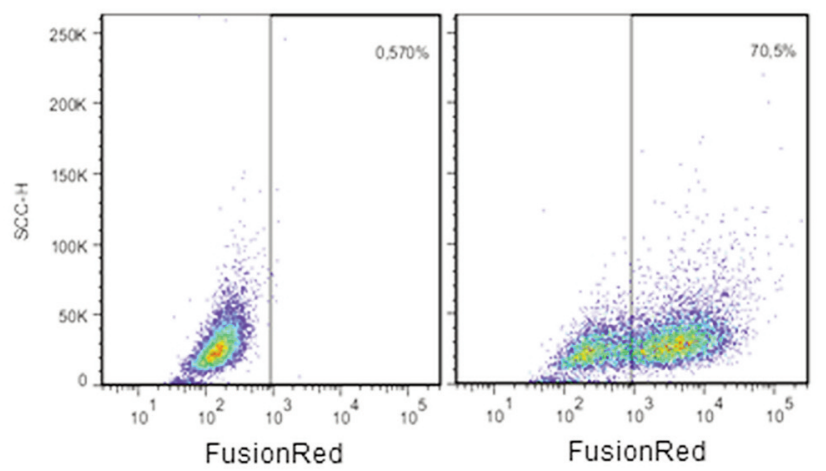

Figure 1. Fluorescence microscopy and flow cytometry analyses of transfected 0840 and 0846 cell lines. (A, B) Merged transmitted light and red fluorescence image of FusionRed expressing cells; FusionRed is localized in the cytoplasm. (C, D) Flow cytometry analyses of FusionRed expression in naive and fluorescent cell lines depicted in dot-plots showing side scatter (SSC) vs. FusionRed fluorescence.

exposure times ( $4 \mathrm{~s}$ and $10 \mathrm{~s}$ ) were used. Images were analyzed with the indiGO software (Berthold Technologies).

Mice used in the animal studies. Mouse studies were approved by the German local authority: Landesamt für Landwirtschaft, Lebensmittelsicherheit und Fischerei Mecklenburg-Vorpommern, according to German animal protection law and EU Guideline

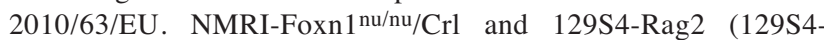
$\operatorname{Rag} 2^{\mathrm{tm} 1.1 \mathrm{Flv}} \mathrm{I} 12 \mathrm{rg}^{\mathrm{tm} 1.1 \mathrm{Flv} / \mathrm{J})}$ mice were originally obtained from Charles River (Rag2 mice were delivered by Charles River in cooperation with Jackson Laboratory) then bred under specific pathogen-free conditions in the animal facility of the University Medical Center in Rostock. Mice were kept in type III cages
(Zoonlab GmbH, Castrop-Rauxel, Germany) at a 12-h dark: light cycle at $21 \pm 2^{\circ} \mathrm{C}$, and humidity of $60 \pm 20 \%$. Cages were enriched by nesting material (shredded tissue paper, Verbandmittel $\mathrm{GmbH}$, Frankenberg, Germany) and a paper roll $(75 \times 38 \mathrm{~mm}, \mathrm{H}$ 0528-151, ssniff-Spezialdiäten $\mathrm{GmbH}$, Soest, Germany). Mice were fed with pellets $(10 \mathrm{~mm}$, ssniff-Spezialdiäten $\mathrm{GmbH})$ and tap water ad libitum. Mouse health, general condition, and distress score were examined three days a week.

Xenograft whole-body imaging. For subcutaneous canine 0840FusionRed or 0846-FusionRed cell injection, two immune-deficient 129S4-Rag2 (female) and two immune-deficient NMRI-Foxn $1 \mathrm{nu} / \mathrm{nu}$ mice (male) were used. Mice were anesthetized by $1.5-2 \%$ 
A
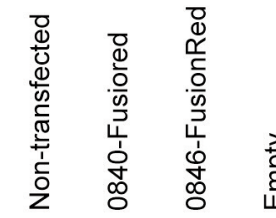

Cell number

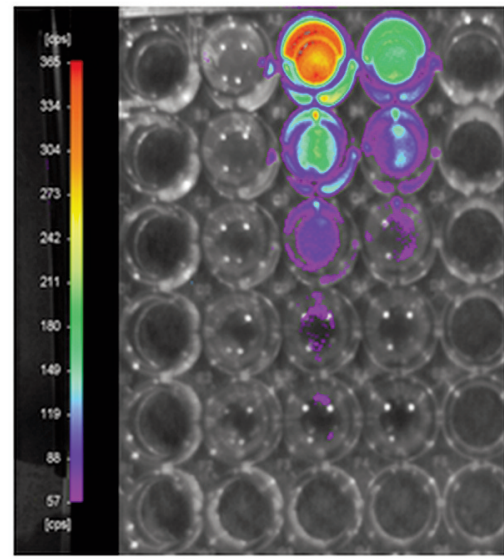

B
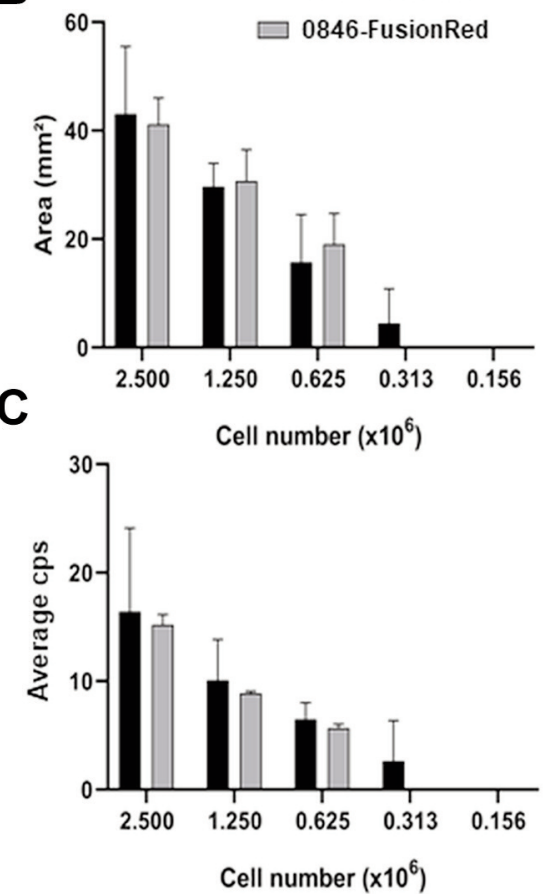

Figure 2. Imaging of transfected 0840 and 0846 cell lines using NightOWL LB983 in vivo imaging system. Serial diluted FusionRed labeled 0840 and 0846 cells were seeded in a 96 well plate overnight. (A) Subsequently, images were taken with NightOWL LB 983 in vivo imaging system using a filter with an excitation at $525 \mathrm{~nm}$ and emission at $655 \mathrm{~nm}$ and $10 \mathrm{~s}$ exposure. Non-transfected cells were used as a negative control. (B) Area $\left(\mathrm{mm}^{2}\right)$ and $(C)$ average counts per second (cps) were evaluated by IndiGO software for both cell lines. Error bars represent the mean \pm standard deviation $(S D)$.

Isoflurane, lower back was shaved and $1 \times 10^{6} 0840$ or 0846 cells were injected subcutaneously in one of each mouse strain in both flanks. For a comparative approach, right flank injection of a 1:2 PBS/Matrigel mixture and left flank injection 100\% 1× PBS were used (Table I). Tumor cell engraftment was assessed by measuring the tumor volume by a caliper and whole-body imaging.

Early engraftment of subcutaneously injected fluorescently labeled canine 0840 or 0846 cells was assessed by whole-body fluorescence imaging using the NightOWL LB 983 System. Engraftment was monitored once a week for a total of five weeks by a $525 \mathrm{~nm}$ GFP excitation and 655 NIR emission filter. To achieve fast recovery from anesthesia, mice were anesthetized for imaging by $1.5-2 \%$ Isoflurane.

RNA isolation and library generation. Total RNA was isolated from transfected and native prostate tumor cell lines using the RNeasy ${ }^{\circledR}$ Mini Kit RNA Purification (Qiagen, Hilden, Germany) according to the manufacturer's instructions. RNA isolation was performed three times per cell line. On-column DNase digestion was carried out with RNase-Free DNase Set (Qiagen) to avoid genomic DNA contamination.

RNA quality was assessed using an Agilent RNA 6000 Nano kit and a 2100 Bioanalyzer (Agilent Technologies Inc., Santa Clara, CA, USA). Samples with RNA integrity numbers (RIN) $>8$ were used for the DNA library preparation using a TruSeq Stranded mRNA Sample Preparation Kit according to the manufacture's protocol (Illumina Inc., San Diego, CA, USA). Briefly, $1 \mu \mathrm{g}$ of total
RNA was used as input for an mRNA enrichment using poly-T oligo coated magnetic beads, and was chemically fragmented under elevated temperature. The fragmented RNA was then reversetranscribed into the first- and second-strand cDNA using random hexamers and Superscript II reverse transcriptase. Double-stranded cDNA fragments were ligated with TruSeq RNA adapters with a unique DNA sequencing index and PCR-amplified. DNA libraries were quality-controlled using an Agilent Technologies 2100 Bioanalyzer and Agilent DNA-1000 Chip kit.

RNA sequencing. cDNA library concentration was quantified using a Qubit dsDNA HS Assay kit (Life Technologies) and normalized to $2 \mathrm{nM}$ before multiplexing. The DNA libraries were sequenced at a final concentration of $13 \mathrm{pM}$ for $125 \mathrm{bp}$ single-end reads using the high-output mode on a HiSeq2500 (Illumina) at the sequencing facility of Genome Biology Institute, Leibniz Institute for Farm Animal Biology, Dummerstorf, Germany.

Data processing and differentially expressed gene (DEGs) analysis. The raw fastq reads were quality-checked using FastQC (version 0.11.5) (22) and pre-processed by filtering out low quality reads with a mean Q-score $<20$ and trimming adapter-like sequences using TrimGalore version 0.6 .5 . High-quality reads were aligned to the reference genome CanFam2 (Ensembl release 100) using Hisat2 version $2.2(23,24)$ Uniquely mapped reads to each gene were extracted from the HISAT2 mapping results using HTSeq version 0.8.0 (25). 
A
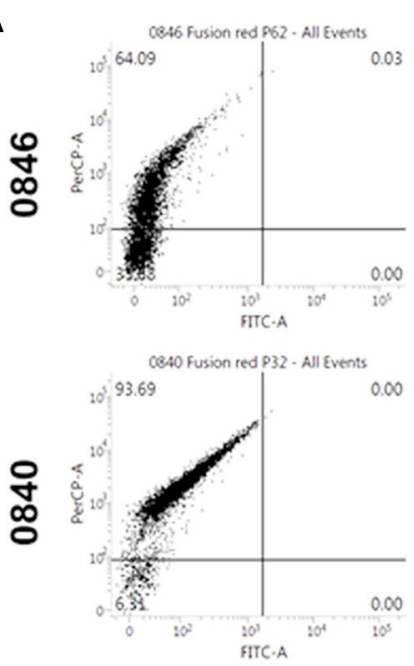

B

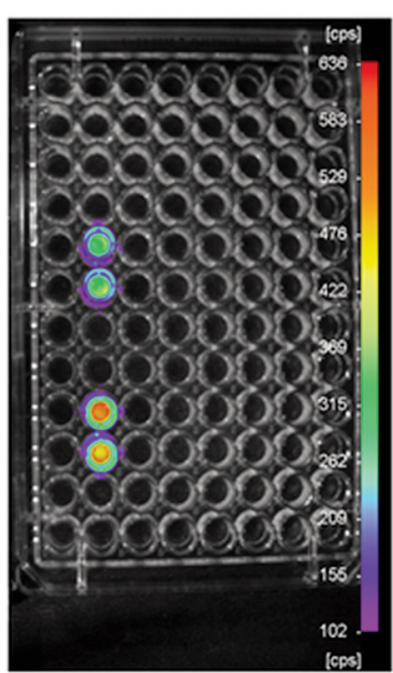

C

Day 2 Day 9

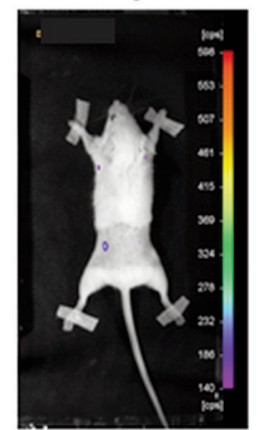

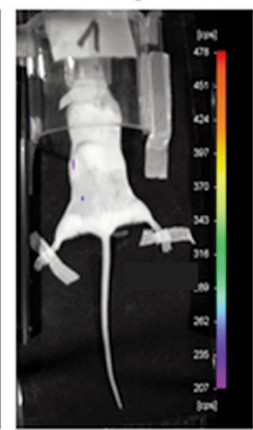

Day 16

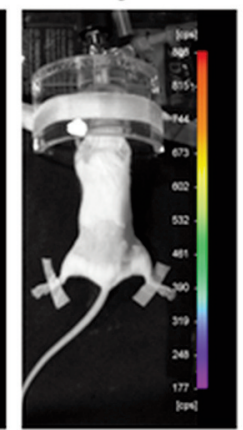

Day 23

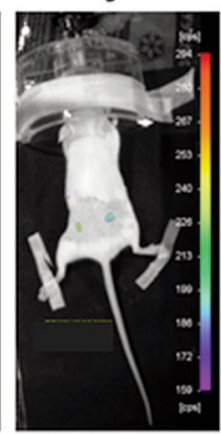

Day 30

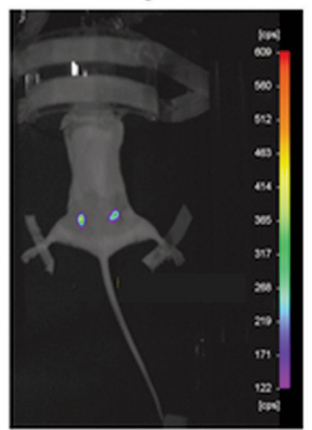

Figure 3. Assessment of fluorescently labeled canine cell early engraftment. Two different FusionRed labeled canine cell lines, 0840 and 0846, were examined regarding their early engraftment ability in the two immune-deficient mouse strains 129S4-Rag2 and NMRI-Foxn1nu/nu. (A) The fluorescent intensity of 0840-FusionRed and 0846-FusionRed cells was examined by flow cytometry. (B) The fluorescent intensity of both cell lines was further examined using NightOWL system. (C) A 129S4-Rag2 mouse (mouse ID: Rag1) was injected with 0840-FusionRed cells and imaged using NightOWL system at the indicated time points after cell injection.

Functional and pathways enrichment analysis. Gene ontology (GO) analysis was applied for the functional annotation and pathway analysis, using the Database for Annotation Visualization and Integrated Discovery (DAVID). The list of all genomes was used as the background parameter.

Data availability statement. RNA-seq data have been deposited in the ArrayExpress database at EMBL-EBI (26) under accession number E-MTAB-10839.

\section{Results}

Generation of red fluorescent tumor cell lines. Transfected cell lines 0840-FusionRed and 0846-FusionRed showed a distinct cytoplasmic red fluorescence 24-72 h posttransfection, whereas native 0840 and 0846 cells showed no FusionRed fluorescence (Figure 1A and B). To quantify the number of FusionRed positive cells, both 0840 and 0846 fluorescent cell lines were compared to native 0840 and 0846 cells by flow cytometry. After 2 months of G418 selection, $95.6 \%$ and $70.5 \%$ of cells were found to be FusionRed positive in 0840-FusionRed and 0846-FusionRed cells, respectively (Figure $1 \mathrm{C}$ and $\mathrm{D}$ ).

In vitro imaging using NightOWL LB 983 in vivo imaging system. Transfected 0840-FusionRed and 0846-FusionRed cells were further imaged in a NightOWL LB 983 in vivo imaging system to illustrate and quantify fluorescent intensity in a whole-body imager. Both cell lines showed comparable cell concentration-dependent fluorescent intensity. The lower limit of detection was $0.3125 \times 10^{6}$ cells per well with average counts per second (cps) of 2.64 \pm 3.734 and $4.485 \pm 6.343 \mathrm{~mm}^{2}$ for 0840 -FusionRed cell line. For 0846 the lower detection 


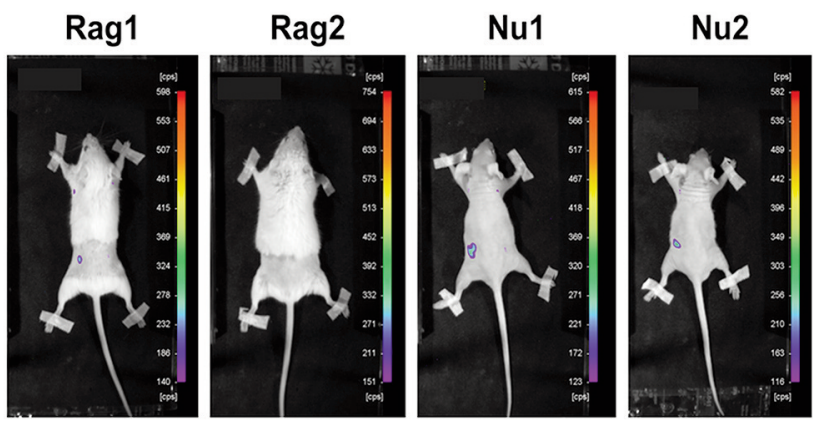

Figure 4. Assessment of fluorescently labeled canine cell engraftment in two different immune-deficient mouse strains. Two different FusionRed labeled canine cell lines, 0840 and 0846, were examined regarding their early engraftment ability in the two immune-deficient mouse strains 129S4-Rag2 and NMRI-Foxn1nu/nu. Images of both cell lines in both mouse strains on day 2 after cell line subcutaneous injection. Early engraftment of 0840-FusionRed cell injected 129S4Rag2 mouse (mouse ID: Rag1), 0846-FusionRed cell injected 129S4Rag2 mouse (mouse ID: Rag2), 0840-FusionRed cell injected NMRIFoxn1nu/nu (mouse ID: Nu1), and 0846-FusionRed injected NMRIFoxn1nu/nu (mouse ID: Nu2).

limit was $0.625 \times 10^{6}$ cells per well with average cps $5.69 \pm 0.339$ and $19.08 \pm 5.629 \mathrm{~mm}^{2}$ (Figure 2).

Early engraftment of fluorescent cell lines is detectable by whole-body imaging. Early engraftment of subcutaneously injected fluorescent canine 0840 or 0846 cells was assessed by whole-body fluorescence imaging using the NightOWL LB 983 System once a week. For control, fluorescent intensity of cells was tested by flow cytometry and wholebody imaging (Figure 3A and B).

0840 cells showed higher fluorescent intensity compared to the 0846 cell line by flow cytometric analyses (0480:

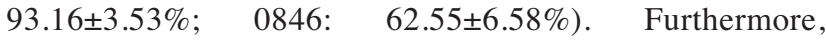
fluorescence imaging by the whole-body imager NightOWL System confirmed higher fluorescent intensity for the 0840 cell line (Figure 3B).

Each cell line was subcutaneously injected in the right flank using a matrigel/PBS mixture and in the left flank using PBS. Injection of the 0840 cell line into the 129S4Rag2 mouse strain showed cell engraftment two days after cell line injection using PBS. On days 9 and 19, the fluorescent signal was not detectable in any of the flanks. However, on days 23 and 30 strong fluorescent intensity for both cell lines was measured by the NightOWL system in right and left flank. 0846-FusionRed cells were not detectable in Rag2 mice over five weeks. NMRI-Foxn1 $\mathrm{nu} / \mathrm{nu}$ mice only showed signal intensity on day 2. 0846FusionRed injected mice (mouse ID: $\mathrm{Nu} 2$ ) lost the signal after two days and 0840-FusionRed injected mice (mouse ID: Nu1) died after day 2 imaging due to anesthesia. Whole-

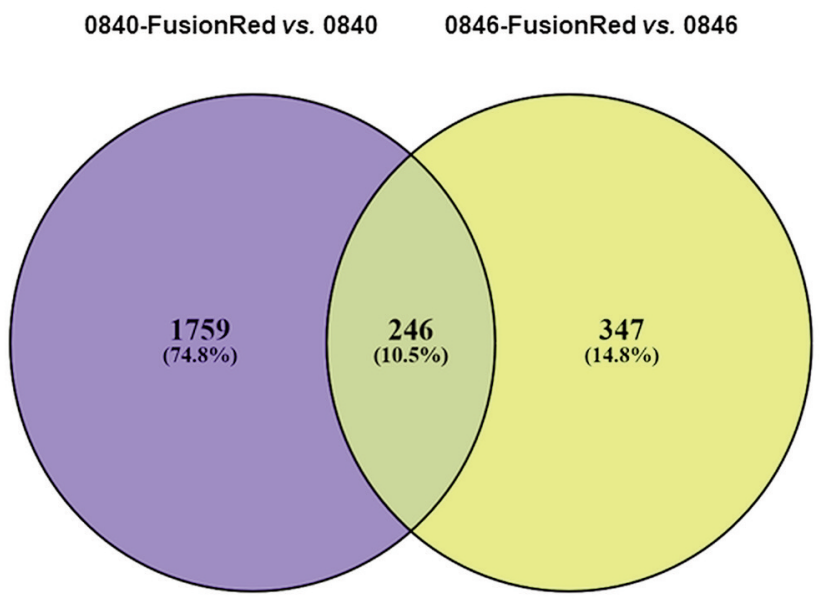

Figure 5. Venn diagram of differentially expressed genes in $0840 \mathrm{vs.}$ 0840-FusionRed and 0846 vs. 0846-FusionRed cells.

body images of both transfected cell lines in both mouse strains on day 2 after injection are displayed in Figure 4.

Comparative analysis of DEGs between transfected and parental cell lines. To investigate, if the introduction of the fusion red gene affected genome stability and gene expression patterns in the transfected cell lines, we compared the transcriptome profiles between the transfected cells and their parental cell lines.

DEGs in the transfected cell lines were identified by thresholds of fold change $\log \mathrm{FC}>1.3$ which corresponds to a fold change of $2.46\left(\log _{2} 2.46=1.3\right)$ and false discovery rate (FDR) $<0.05$ when compared to non-transfected parental cells. A total of 2005 DEGs were found between 0840FusionRed and 0840 cells. Whereas the comparison between 0846-FusionRed and 0846 revealed 593 DEGs. The majority of the DEGs in 0840-FusionRed cells (74.8\% of 2005 genes) were not found deregulated in the 0846-FusionRed cell line. Further, 346 of the DEGs in 0846-FusionRed cells were exclusive to these cells. A total of 246 DEGs were shared among the transfected cell lines (Figure 5).

Functional and pathway enrichment analysis. Enriched pathways and functional classification analysis of DEGs in transfected cell lines were performed using DAVID online tool. The GO analysis of genes expressed in 0840-FusionRed and 0846-FusionRed cells compared to their parental cells revealed enrichment of DEGs for several cellular components (CC) related to the integral component of the membrane and the extracellular space (Table II and Table III).

Molecular function (MF) analysis indicated that the DEGs were only associated with calcium ion binding in 0840FusionRed cells, and calcium ion binding and chemokine 
Table II. GO analysis of genes expressed in 0840-FusionRed cells vs. 0840-FusionRed cells.

\begin{tabular}{lccc}
\hline ID & Term & Count & FDR \\
\hline Cellular components & & 347 & 0.000000000894 \\
GO:0016021 & Integral component of membrane & 0.00000000202 \\
GO:0005887 & Integral component of plasma membrane & 0.00000242 \\
GO:0009986 & Cell surface & 59 & 0.000521 \\
GO:0005615 & Extracellular space & 88 & 0.00531 \\
GO:0005578 & Proteinaceous extracellular matrix & 23 & 0.0202 \\
GO:0005604 & Basement membrane & 13 & 0.0135 \\
GO:0005576 & Extracellular region & 26 & 0.000752 \\
GO:0009897 & External side of plasma membrane & 0.0202 \\
GO:0016324 & Apical plasma membrane & 28 & 26 \\
Molecular functions & Calcium ion binding & 68 & 0.00556 \\
GO:0005509 & & & \\
\hline
\end{tabular}

FDR: False discovery rate.

Table III. Gene ontology analysis of genes expressed in 0840-FusionRed cells vs. 0840-FusionRed cells.

\begin{tabular}{lccc}
\hline ID & Term & Count & FDR \\
\hline Cellular components & & 47 & 0.000000129 \\
GO:0005615 & Extracellular space & 96 & 0.0294 \\
GO:0070062 & Extracellular exosome & 13 & 0.00160 \\
GO:0005578 & Proteinaceous extracellular matrix & 18 & 0.0000147 \\
GO:0005576 & Extracellular region & 36 & 0.0000634 \\
GO:0005887 & Integral component of plasma membrane & 112 & 0.00160 \\
GO:0016021 & Integral component of membrane & 14 & 0.00471 \\
GO:0016324 & Apical plasma membrane & & \\
Molecular functions & & 28 & 0.0170 \\
GO:0005509 & Calcium ion binding & 6 & 0.0170 \\
GO:0008009 & Chemokine activity & \\
\hline
\end{tabular}

FDR: False discovery rate.

activity in 0846-FusionRed cells (Figure 6). Further, the GO Term analysis demonstrated no enrichment of DEGs for the Biological process (BP) (data not shown).

Thus, the integration of the FusionRed gene into the genome of these cells changed the expression of cell membrane proteins but had no effects on crucial biological processes such as cell proliferation or cell death.

\section{Discussion}

In vivo models are the key to understand the pathogenesis of prostate cancer and develop novel therapeutic approaches (2). Although in vitro systems offer several possibilities for basic drug evaluation, they remain limited for the evaluation of complex interactions. The use of proteins emitting longwavelength fluorescence to label cancer cells enables deep tissue imaging; thereby allowing real-time tracing of cancer growth, metastasis, and determination of the efficacy of candidate antitumor and anti-metastatic therapies. An optimal fluorescent protein for whole-body imaging should have excitation and emission spectra within 650-950 nm. This "optical window" has the lowest absorbance by hemoglobin, melanin, and water in mammalian tissue (9).

In the current study, two canine cancer cell lines, stably expressing a red fluorescent protein, were established. In 0840-FusionRed, more than $90 \%$ of cells were FusionRed positive and able to maintain a considerable fluorescent expression level after long-term cultivation event without selective antibiotic pressure. 0846-FusionRed cells represent a polyclonal transfected cell line, and after long-term G418 selection, $70.5 \%$ of cells expressed FusionRed protein. The expression of red fluorescence protein was also easily detected by NightOWL LB 983 in transfected 0840 and 0846 cell lines (detection of $0.312 \times 10^{6}$ and $0.625 \times 10^{6}$ of $0840-$ FusionRed and 0846-FusionRed respectively) in vivo. Thus, our data as well as those from other publications demonstrate 

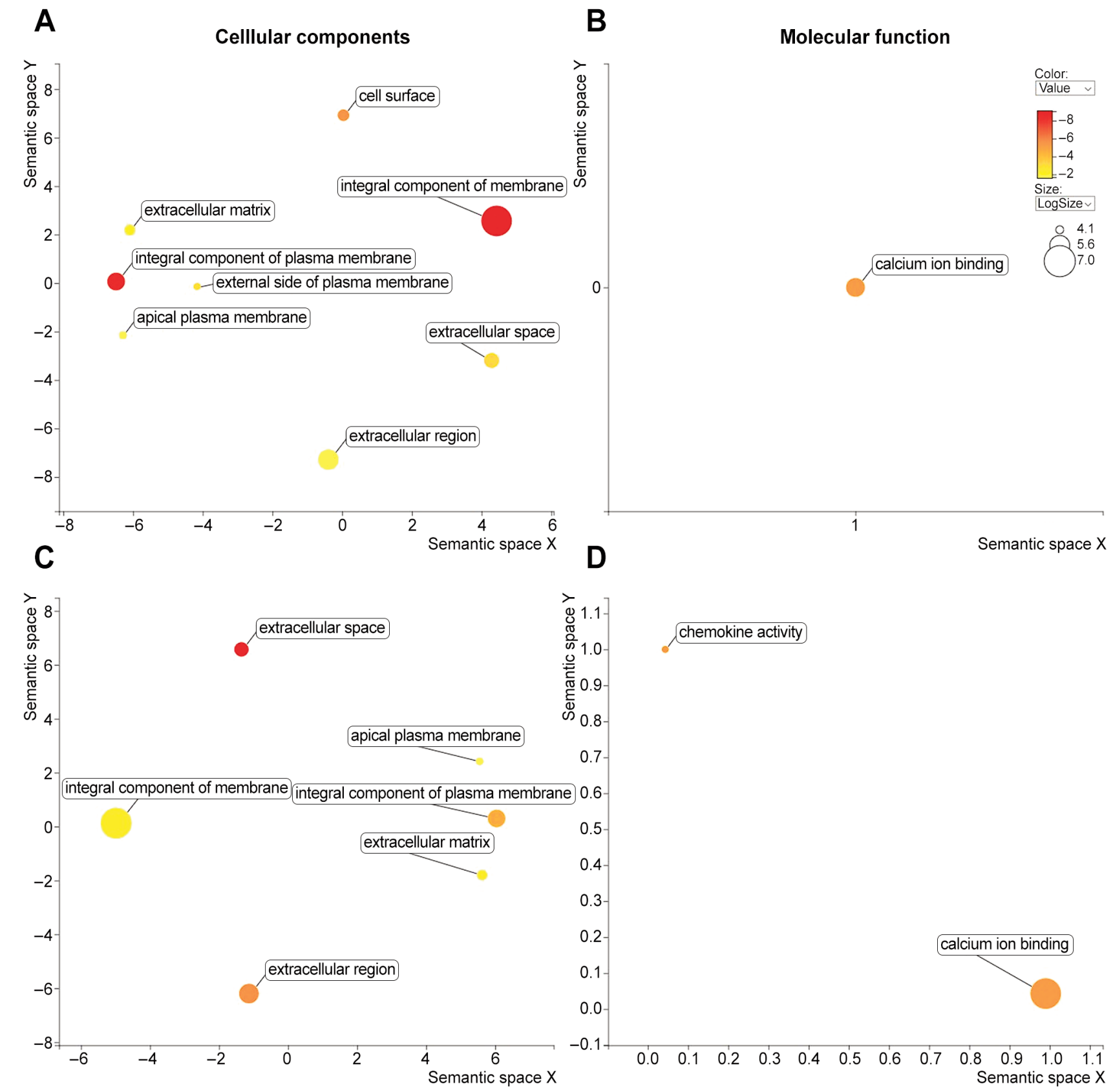

Figure 6. Gene ontology process: common cellular component and molecular function of differentially expressed genes (DEGs) in transfected cell lines. (A) Cellular component of DEGs in 0840-FusionRed cells, (B) molecular function of DEGs in 0840-FusionRed cells, (C) cellular component of DEGs in 0846-FusionRed cells, (D) molecular function of DEGs in 0846-FusionRed cells.

that stably transfected cell lines can provide an excellent tool for long-term imaging and monitoring longitudinal tumor development, for example, for assessing drug response (27). Stable integration of DNA can affect the genome stability of cell lines, therefore, transcriptome analysis of transfected cell lines in comparison to non-transfected cells was conducted to evaluate, if the genomic integration of the transgene has a significant impact on gene expression (28). 0840FusionRed cells revealed more DEGs (2005) in comparison to the 0846-FusionRed cells (593). The difference could be explained by the percentage of FusionRed positive cells, where $70 \%$ of 0846 -FusionRed cells were positive for 
FusionRed and therefore showed few DEGs in comparison to the parental line, whereas more than $90 \%$ of 0840 FusionRed cells expressed FusionRed protein, the background of non-transfected cell lines in 0846 may reduce the number of DEGs. Nevertheless, no significant changes in the biological process of the transfected cells were detected. Further, the genome profiling of 0840-FusionRed and 0846-FusionRed cells in comparison to their parental cells showed significant changes in the expression of cellular components and molecular function but no significant changes in biological processes. The analysis of individual genes revealed that often individual genes were found in multiple categories of cellular components and molecular functions. The introduction of FusionRed did not influence biological processes in the generated cell lines. Consequently, key aspects of the generated cell lines remained comparable to the parental line. In conclusion, the in vivo imaging proved that fluorescent canine prostate adenocarcinoma and transitional cell carcinoma cells were able to develop a detectable tumor in immune deficient mice. Therefore, the generated cell lines represent a valuable tool for in vivo evaluation of novel therapeutic approaches.

\section{Conflicts of Interest}

The Authors declare that they have no competing interests in relation to this study.

\section{Authors' Contributions}

Conceptualization, I.N., C.J and H.M.E.; Methodology, S.A., D.Z., B.V., I.N. and H.M.E; Validation, S.A., S.S. and N.T.; Formal Analysis, S.A., S.S. and N.T..; Investigation, S.A, S.S., S.V.P, and N.T.; Resources, I.N., D.Z., and H.M.E; Data Curation, N.T.; Writing - Original Draft Preparation, S.A.; Writing - Review \& Editing, J.T.S., I.N., D.Z., S.S., N.T. and H.M.E; Visualization, S.A.; Supervision, I.N. and H.M.E.; Project Administration, S.A.; Funding Acquisition, I.N.

\section{Acknowledgements}

The Authors thank Tina Kostka for transfection of the 0846 cell line. Further, the Authors wish to thank Aleppo University (Aleppo, Syria) and HGFK (Hannoversche Gesellschaft zur Förderung der Kleintiermeidizin e.V, (Hannover, Germany) for supporting SA with a scholarship.

\section{References}

1 Rütgen BC, Willenbrock S, Reimann-Berg N, Walter I, FuchsBaumgartinger A, Wagner S, Kovacic B, Essler SE, Schwendenwein I, Nolte I, Saalmüller A and Murua Escobar H: Authentication of primordial characteristics of the CLBL-1 cell line prove the integrity of a canine B-cell lymphoma in a murine in vivo model. PLoS One 7(6): e40078, 2012. PMID: 22761949. DOI: $10.1371 /$ journal.pone. 0040078
2 Cunningham D and You Z: In vitro and in vivo model systems used in prostate cancer research. J Biol Methods 2(1): e17, 2015. PMID: 26146646. DOI: 10.14440/jbm.2015.63

3 Hoffman RM: The multiple uses of fluorescent proteins to visualize cancer in vivo. Nat Rev Cancer 5(10): 796-806, 2005. PMID: 16195751. DOI: 10.1038/nrc1717

4 Hoffman RM and Yang M: Whole-body imaging with fluorescent proteins. Nat Protoc 1(3): 1429-1438, 2006. PMID: 17406431. DOI: 10.1038 /nprot.2006.223

5 Shcherbakova DM and Verkhusha VV: Near-infrared fluorescent proteins for multicolor in vivo imaging. Nat Methods 10(8): 751754, 2013. PMID: 23770755. DOI: 10.1038/nmeth.2521

6 Luker KE, Pata P, Shemiakina II, Pereverzeva A, Stacer AC, Shcherbo DS, Pletnev VZ, Skolnaja M, Lukyanov KA, Luker GD, Pata I and Chudakov DM: Comparative study reveals better far-red fluorescent protein for whole body imaging. Sci Rep 5: 10332, 2015. PMID: 26035795. DOI: 10.1038/srep10332

7 Hoffman R: The advantages of using fluorescent proteins for in vivo imaging. Current Protocols Essential Laboratory Techniques 15(1), 2020. DOI: $10.1002 /$ cpet.12

8 Shcherbo D, Merzlyak EM, Chepurnykh TV, Fradkov AF, Ermakova GV, Solovieva EA, Lukyanov KA, Bogdanova EA, Zaraisky AG, Lukyanov S and Chudakov DM: Bright far-red fluorescent protein for whole-body imaging. Nat Methods 4(9): 741-746, 2007. PMID: 17721542. DOI: 10.1038/nmeth1083

9 Shcherbakova DM, Subach OM and Verkhusha VV: Red fluorescent proteins: advanced imaging applications and future design. Angew Chem Int Ed Engl 51(43): 10724-10738, 2012. PMID: 22851529. DOI: 10.1002/anie.201200408

10 Jacobsen L, Calvin S and Lobenhofer E: Transcriptional effects of transfection: the potential for misinterpretation of gene expression data generated from transiently transfected cells. Biotechniques 47(1): 617-624, 2009. PMID: 19594446. DOI: 10.2144/000113132

11 Reis-Filho JS: Next-generation sequencing. Breast Cancer Res 11 Suppl 3: S12, 2009. PMID: 20030863. DOI: 10.1186/bcr2431

12 Waters DJ, Sakr WA, Hayden DW, Lang CM, McKinney L, Murphy GP, Radinsky R, Ramoner R, Richardson RC and Tindall DJ: Workgroup 4: spontaneous prostate carcinoma in dogs and nonhuman primates. Prostate 36(1): 64-67, 1998. PMID: 9650919. DOI: 10.1002/(sici)1097-0045(19980615)36: $1<64::$ aid-pros $12>3.0 . c 0 ; 2-\mathrm{g}$

13 Palmieri C, Foster RA, Grieco V, Fonseca-Alves CE, Wood GA, Culp WTN, Murua Escobar H, De Marzo AM and LauferAmorim R: Histopathological terminology standards for the reporting of prostatic epithelial lesions in dogs. J Comp Pathol 171: 30-37, 2019. PMID: 31540623. DOI: 10.1016/j.jcpa. 2019.07.005

14 Leroy BE and Northrup N: Prostate cancer in dogs: comparative and clinical aspects. Vet J 180(2): 149-162, 2009. PMID: 18786842. DOI: $10.1016 /$ j.tvj1.2008.07.012

15 Sun F, Báez-Díaz C and Sánchez-Margallo FM: Canine prostate models in preclinical studies of minimally invasive interventions: part I, canine prostate anatomy and prostate cancer models. Transl Androl Urol 6(3): 538-546, 2017. PMID: 28725597. DOI: $10.21037 /$ tau.2017.03.61

16 Lai CL, van den Ham R, van Leenders G, van der Lugt J, Mol JA and Teske E: Histopathological and immunohistochemical characterization of canine prostate cancer. Prostate 68(5): 477488, 2008. PMID: 18196537. DOI: 10.1002/pros.20720 
17 Cekanova M and Rathore K: Animal models and therapeutic molecular targets of cancer: utility and limitations. Drug Des Devel Ther 8: 1911-1921, 2014. PMID: 25342884. DOI: 10.2147/DDDT.S49584

18 Reimann-Berg N, Willenbrock S, Murua Escobar H, Eberle N, Gerhauser I, Mischke R, Bullerdiek J and Nolte I: Two new cases of polysomy 13 in canine prostate cancer. Cytogenet Genome Res 132(1-2): 16-21, 2011. PMID: 20668368. DOI: $10.1159 / 000317077$

19 Packeiser EM, Hewicker-Trautwein M, Thiemeyer H, Mohr A, Junginger J, Schille JT, Murua Escobar $\mathrm{H}$ and Nolte I: Characterization of six canine prostate adenocarcinoma and three transitional cell carcinoma cell lines derived from primary tumor tissues as well as metastasis. PLoS One 15(3): e0230272, 2020 PMID: 32168360. DOI: 10.1371/journal.pone.0230272

20 Harting T, Stubbendorff M, Willenbrock S, Wagner S, Schadzek P, Ngezahayo A, Murua Escobar HM and Nolte I: The effect of dichloroacetate in canine prostate adenocarcinomas and transitional cell carcinomas in vitro. Int J Oncol 49(6): 23412350, 2016. PMID: 27748833. DOI: 10.3892/ijo.2016.3720

21 Hammer SC, Nagel S, Junginger J, Hewicker-Trautwein M, Wagner S, Heisterkamp A, Ngezahayo A, Nolte I and Murua Escobar H: Claudin-1, -3, -4 and -7 gene expression analyses in canine prostate carcinoma and mammary tissue derived cell lines. Neoplasma 63(2): 231-238, 2016. PMID: 26774145. DOI: 10.4149/208_150924N505

22 Babraham Bioinformatics. Available at: http://www.bioinformatics. babraham.ac.uk/projects/fastqc/ [Last accessed on November 7 , 2021]
23 Kim D, Langmead B and Salzberg SL: HISAT: a fast spliced aligner with low memory requirements. Nat Methods 12(4): 357360, 2015. PMID: 25751142. DOI: 10.1038/nmeth.3317

24 Pertea M, Kim D, Pertea GM, Leek JT and Salzberg SL: Transcript-level expression analysis of RNA-seq experiments with HISAT, StringTie and Ballgown. Nat Protoc 11(9): 16501667, 2016. PMID: 27560171. DOI: 10.1038/nprot.2016.095

25 Anders S, Pyl PT and Huber W: HTSeq-a Python framework to work with high-throughput sequencing data. Bioinformatics 31(2): 166-169, 2015. PMID: 25260700. DOI: 10.1093/ bioinformatics/btu638

26 ArrayExpress-functional genomics data. Available at: www.ebi.ac.uk/arrayexpress [Last accessed on November 7, 2021]

27 Liang L, Yue Z, Du W, Li Y, Tao H, Wang D, Wang R, Huang Z, He N, Xie X, Han Z, Liu N and Li Z: Molecular imaging of inducible VEGF expression and tumor progression in a breast cancer model. Cell Physiol Biochem 42(1): 407-415, 2017. PMID: 28558368. DOI: 10.1159/000477485

28 Hardee CL, Arévalo-Soliz LM, Hornstein BD and Zechiedrich $\mathrm{L}$ : Advances in non-viral DNA vectors for gene therapy. Genes (Basel) 8(2): 65, 2017. PMID: 28208635. DOI: 10.3390/ genes8020065
Received September 30, 2021

Revised November 7, 2021

Accepted November 14, 2021 